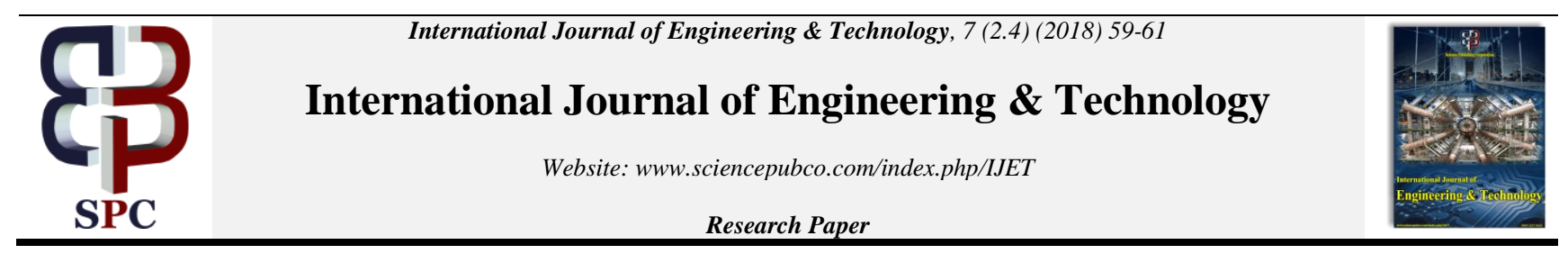

\title{
High Mobility Supports Routing Protocol (HMSRP) in Mobile Ad Hoc Networks
}

\author{
Dr.T.Senthil Murugan \\ ${ }^{I}$ Research Scholar, Veltech Rangarajan Dr. Sagunthala R\&D Institute Science \& Technology, Chennai. \\ ${ }^{2}$ Associate Professor, Department of Computer Science \& Engineering, \\ Veltech Rangarajan Dr. Sagunthala R\&D Institute Science \& Technology, Chennai \\ Email: senthilmuruganme@gmail.com
}

\begin{abstract}
Now-a-days wireless communication required for infrastructure less environment due to avoiding centralized data maintenance and fixed infrastructure data transmission because these types of network not supporting in emergency situation like natural disaster, Battle field and so on. But, Mobile Ad-Hoc networks (MANETs) are support the data communication in the above mentioned situations. In MANET, network implementation and maintenance has many emerging research areas like routing, Energy management, Quality of services, etc. Even many types of protocols are proposed and identified in this area but still it is more complex for identifying quality route. One of the most important factors for identifying quality route is "Mobility". Many researchers are proposed protocols based on routing with random mobility. In this paper, we propose a new routing protocol "High Mobility Supports Routing Protocol (HMSRP)" which is supporting high mobility. Mobility has to be calculated using directional antennas. This proposed new protocol has supports to identify the location of the node, enhance the fast data transmission and control the fast communication. Simulation results are shown our proposed protocol is produce good results over other protocol.
\end{abstract}

\section{Introduction}

Basically Mobile Ad Hoc network is infrastructure less network means there is no centralized server or maintenance were nodes are configured itself and establish a communication link with each other. Communication establishing may be direct to particular node which is available in communication range of the node or indirectly communicate with the help of other nodes which is participated in the network.

MANET is used to monitoring surveillance the real time environments, inspect the power transmitting electrical lines, flood rescue operation, monitoring the forest like animal movements, firing in forest, make communication at Earthquake and Tsunami, etc. In Emergency situations, making infrastructure network is complicate. But MANT support the communication were mobile nodes are acting as a router. All the participants nodes of the this network is acting as a router for making connection between source to destination using routing table and transmitting the data through this routers (intermediate nodes).

MANET routing protocols are broadly classified into table driven routing protocol (Proactive) and On-demand routing protocol (Reactive). These protocols basically support to identify the route from source to destination. Based on the maintaining routing table its may classified. Table driven routing methods maintaining the routing table which is contains all information of other nodes.
Routing table helps to select the path for establishing connection between sources to destination. Rather than maintaining entire information of other nodes in routing table, to be identify a route in on demand basis is done by on-demand routing protocol.

Nodes movement has to be updated in routing table with the help of the global positioning systems (GPS) which is providing the location of the node. Directional antennas are producing the positions of the node even the nodes are moving with high mobility without depending other nodes and any directional.

\section{Related Works}

DaehoKang et al. [1] proposed a protocol which support reliably and practically opportunistic. They applied signal to noise ratio for maintaining light weight routing management. Its produce good results close to an perfect routing with total information.

Mingchuan Zhang et al. [2] presented a hybrid protocol which is combined with ant colony optimization, assessments of trust and physarum autonomic optimization. As a first step, they divided the networks into several zones. The zones are maintaining routing table by perspective ants as a proactive method. These ants are find a routes as reactive method then send to the destination. In mean time, the proposed protocol is chosen a optimized path using sense parameters.

Philipp H. Kindt et al. [3] proposed a mathematical theory for identifying latency of packets reached by neighbor node with all 
the required parameters. Many of the protocols using slotted methods for calculate the latency and recent protocols are using slot less methods which is compute periodical basis based on advertising intervals, Scanning process and duration of the scanning process. The mathematical model which is proposed by author is finding out the maximum bound level of latency with all types of parameterization and its helps to analysis the latency for future developing protocols.

Aqeel Taha et al. [4] proposed a multipath routing protocol in MANET which is used fitness function for managing energy of the nodes which is participated in networks. The mobile nodes are moving or changing their position time to time. Based on high mobility, the nodes are not able to charging their batteries. Hence the fitness function helps to maintain the energy of the node. They compared the results between existing and proposed protocols.

Waheb A.Jabbar et al. [5] have simulated a new multipath routing protocol which is support battery stability and faster mobility of nodes. These two factors are important for improving the performance of the entire network. They used a term Multi criteria node rank for compute the residual energy of the node as well as predicting the mobility speed and give a rank for the route which have more stability with the help of link assessment function. Pitchaimuthu Francis Antony Selvi and Moola Seetharamaiyer Kasiviswanathan Manikandan proposed a protocol in MANET which is support load balancing multiple paths. This method using swarm based ant colony optimization to skip fault routes for finding better route and also concentrate the load balancing of the route like distributing the load.

\section{Proposed Methodology}

MANET is mainly used for emergency situation like natural disaster, battle field and so on. Many routing protocols have proposed in mobile ad hoc network for enhancing the quality of the services of the network. All proposed protocols are considered and simulated using network simulators. Hence the routing methods are considering only energy as battery power and delay of the route for finding shortest path. Our proposed routing algorithm concentrates to identifying best route with high mobility neighbor node. It's also concentrate both energy and delay with using LSEA routing [7] algorithm.

\subsection{High Mobility Supports Routing Protocol (HMSRP)}

//Discovery of position of a node//

(1)If (Node== Sender), its sends the data packets with the following information

(i)Position vector of GPS to be updated in request packets by each node

(ii)Updating the status of transmitting data by themselves

(iii)Getting the transmission range of directional antenna and update in packets

(2)If (Node $==$ Neighbor) // Node with in sender communication range

(i)Initialize listening mode

(ii)Stop and reschedule the current transmitting packets

(iii)Update the information about themselves in sender packet

// Sender calculate the correct position of the neighbor//

(3)Sender (Calculating the position of the neighbor nodes)

(i)mobility like present position (getting from GPS)

(ii)movement speed (in Velocity) at current time
//Routing packets maintenance //

(4)Sender send request permission for forwarding packets to neighbor

If( neighbor is free and ready to receive)

Send Route Clear packet to sender

// neighbor is busy

else Send waiting message to sender

(5)Neighbor is ready to receive the packets //Data Transmission

Sender getting current position of the node using directional antenna

Forward the packets towards directional antenna with best energy nodes

//best energy nodes be identified using LESA algorithm

If (node moving with high speed)

go to (step 3)

\subsection{Route establishment:}

Link stability and energy aware (LSEA) routing protocols helps to identify best quality route which is consider stability of link and remaining energy of the intermediate nodes. Additionally, the proposed HMSRP is identifying directions of fast moving nodes with the help of directional antenna and transmitting control messages like ready, wait and busy. After getting ready signal and direction from the neighbor node, a route has established. Directional antenna and GPS are using for location of the node. The proposed algorithm is predicting the accurate location of the node with the help of Location which is shared by GPS and velocity of the node and its take only extra 17 bytes.

\subsection{Data Transmission:}

The important task of this protocol is decision making and optimizing itself without human interaction. In decision making phase, a node takes decision about how to transfer a packet with which neighbor nodes to the destination. In self optimizing phase, this protocol selects a shortest path with less time. Hence, ultimately reduce the delay of packet delivery. This protocol significantly increases the data transmission over the network.

\section{Results and Discussion:}

The algorithm is simulated in network simulator environments with the following parameter and values. Simulated results has illustrated in graphs for better understanding. Graphical representations is projected the results of simulation and project the proposed protocols is better than existing protocols.

\begin{tabular}{|l|l|}
\hline Parameter & Value \\
\hline Dimensions & $1000 \times 1000 \mathrm{~m}^{2}$ \\
\hline Number of nodes & 25 \\
\hline Simulation time & $100 \mathrm{~s}$ \\
\hline Packet size & 512 bytes \\
\hline MAC Layer & IEEE $802.11 \mathrm{~b}$ \\
\hline Maximum speed & $20 \sim 100 \mathrm{~m} / \mathrm{s}$ \\
\hline Pause time & $10 \mathrm{~s}$ \\
\hline Connection Rate & $3 \mathrm{Packets} / \mathrm{Sec}$ \\
\hline
\end{tabular}




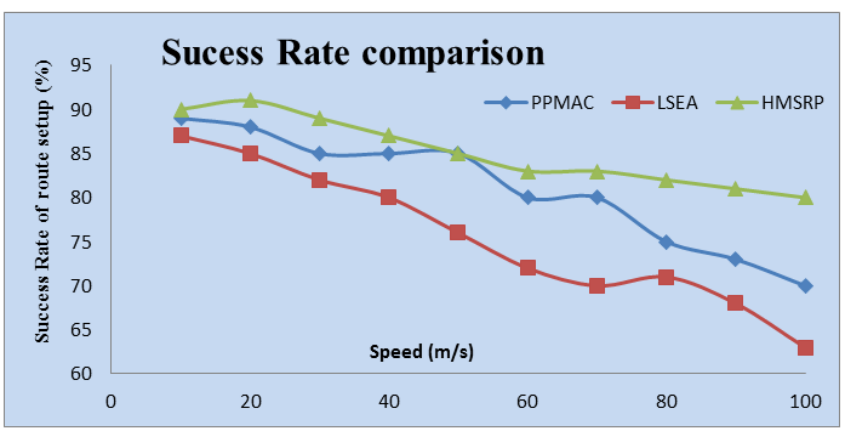

Fig. 1 Success Rate in $\%$ of route setup

In Fig.1, The proposed HMSRP algorithm maintains above $80 \%$ of success rate for forming the best routes compared with LSEA and PPMAC algorithm. Increasing of node mobility is to reduce the success rate of route setup. But the proposed protocols have maintained the stability of the link and increasing the success rate of route setup.

Fig. 2 represents the life time of the route which is used for data transmission over the ad hoc network. Here, number of the nodes participated in data transmission is also the factor for route life time. If a node have less energy and the particular node involved in data transmission than after some time the particular node will get shutdown. Hence entire route will be spoiled. Due to increasing the life time of the route, this proposed protocol has considered the remaining energy of the individual nodes and permit the nodes for data transmission.

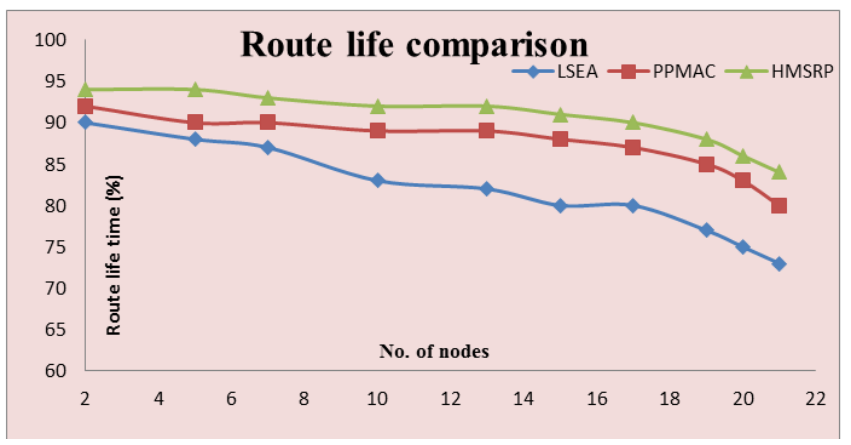

Fig.2 Life time of the route

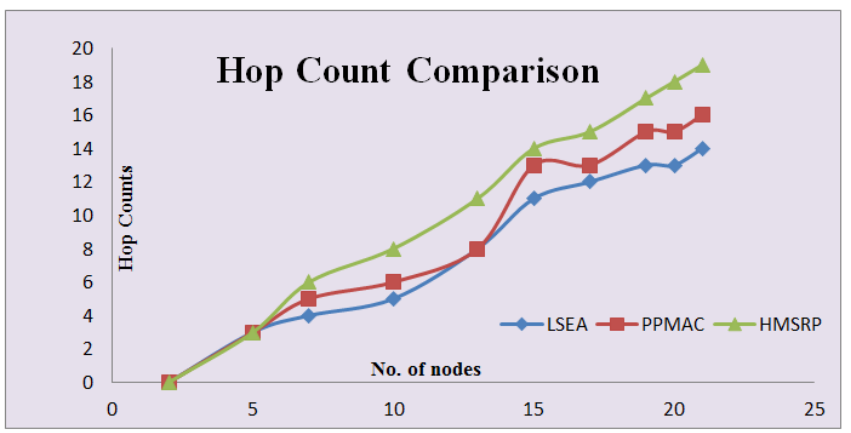

Fig.3 Hop Count Vs Number of nodes

In fig.3, Hop count is compared with some routing protocols in view of increasing the number of nodes. In minimum number of nodes, all three protocols have maintained same number of hop counts. If increasing the number of nodes in the network, the hop count also increasing due to identified multiple routes with the quality manner and selecting shortest route. In this point of view, the proposed protocol maintains the maximum number of hop counts compared with other two protocols.

\section{Conclusion:}

The proposed HMSRP algorithm is suitable for high speed mobility ad hoc network. Its shows better results compared with existing protocols like LSEA and PPMAC. This proposed protocols have some little bit of extra overload due to maintain the positions, speed, energy and delay. Even its have this overloads, its produced good results which is illustrated in graphs. This protocol has suffered the quality factor delay. Hence, its compute fast \& long life time route and its predict the position of the node for casting the data without loss. Finally, I conclude this protocol is better than other protocols and suitable for fast moving networks.

\section{References}

[1] DaehoKang, Hyung-SinKim, ChangheeJoo, Saewoong Bahk, "ORGMA: Reliable opportunistic routing with gradient forwarding for MANETs", Elsevier - Computer Networks, 131, 2018, 52-64.

[2] Mingchuan Zhang, Meiyi Yang Qingtao, Wu Ruijuan Zheng, Junlong Zhu, "Smart perception and autonomic optimization: A novel bio-inspired hybrid routing protocol for MANETs", Elsevier - Future Generation Computer Systems, 81, 2018, 505513.

[3] Philipp H. Kindt, Marco Saur, Michael Balszun, "Neighbor Discovery Latency in BLE-Like Protocols", IEEE Transactions on Mobile Computing, 17 (3), 2018, $617-631$.

[4] Aqeel Taha, Raed Alsaqour, Mueen Uddin, Maha Abdelhaq, Tanzila Saba, "Energy Efficient Multipath Routing Protocol for Mobile Ad-Hoc Network Using the Fitness Function", IEEE Access, 5, 2017, $10369-10381$.

[5] Waheb A.Jabbar, Mahamod Ismail, Rosdiadee Nordin, "Energy and mobility conscious multipath routing scheme for route stability and load balancing in MANETs", Elsevier - Simulation Modelling Practice and Theory, 77, 2017, 245-271.

[6] Pitchaimuthu Francis Antony Selvi, Moola Seetharamaiyer Kasiviswanathan Manikandan, "Ant based multipath backbone routing for load balancing in MANET", IET Communications, $11,(1), 2017,136-141$.

[7] Senthil Murugan Tamilarasan and Kannan Eswariah "Link Stability with Energy Aware Ad Hoc On Demand Multipath Routing Protocol In Mobile Ad Hoc Networks" American Journal of Applied Sciences 10 (8), 2013, 844-848. 